\title{
Propagation study of UWB capsule endoscope with multiple on-body antennas
}

\author{
Mariella Särestöniemi \\ Center for Wireless Communications, \\ Faculty of Information Technology and \\ Electrical Engineering \\ University of Oulu \\ Oulu, Finland \\ mariella.sarestoniemi@oulu.fi
}

Jari Iinatti

Center for Wireless Communications,

Faculty of Information Technology and

Electrical Engineering

University of Oulu

Oulu, Finland

jari.iinatti@oulu.fi

\author{
Carlos Pomalaza Raez \\ Department of Electrical and Computer \\ Engineering \\ Purdue University \\ Fort Wayne, Indiana 46805, USA \\ cpomalaz@purdue.edu
}

\author{
Chaïmaâ Kissi \\ Electronics and Telecommunication \\ Systems Research Group, National \\ School of Applied Sciences (ENSA) \\ Ibn Tofail University \\ Kenitra, Morocco \\ chaimaakissi1@gmail.com
}

\begin{abstract}
This paper presents a study of the radio channel characteristics between a capsule endoscopy and a multi onbody antenna system on ultra wideband wireless body area networks (UWB-WBAN). Multiple on-body antennas are required to provide reliable communication link between the capsule and the on-body device, but also essential for capsule localization. The main aim is to study the variation of the frequency and time domain channel characteristics for the selected on-body antennas in different capsule locations, including the most challenging capsule locations deep inside the tissues or far away from most of the antennas. This study also evaluates whether five of selected type directive on-body antennas is enough to cover the intestine area thoroughly. The study is conducted with CST Studio Suite simulations and one of its anatomical voxel models. A simplified capsule model and a directive on-body antenna designed for low-band UWB inbody communications are used in this study. It is found that five of this type directive on-body antennas provide sufficient coverage over the intestine area even in the most challenging capsule locations. In certain capsule locations, the variation between the channel attenuations can be significant, over $40 \mathrm{~dB}$ within the frequency range of interest, if the capsule is located deep inside the tissues without smooth access to outer fat layer through which the signal could travel easily to different on-body antennas. Instead, if the capsule is located close to the subcutaneous fat layer, the channel attenuation is moderate even for the antennas which are located far from the capsule.
\end{abstract}

Keywords-antenna gain, directive on-body antenna, capsule endoscope, fat propagation medium, multiantenna system, ultra wideband, wireless body area networks.

\section{INTRODUCTION}

Capsule endoscopy, which is a non-invasive, painless, and reliable method to investigate the small intestine thoroughly, has become an actively investigated topic [1]-[4]. Recently it has been recognized that ultra wideband (UWB) technology could provide several advantages for capsule endoscopy, such as high resolution images, high data rate, low power, reliability [4]. However, one of the main challenges related to UWB based capsule endoscopy, or in general UWB implant communications, is the high propagation loss in the tissues [5].
Propagation characteristics between the capsule and the onbody antenna has been actively studied topic e.g. in [6]-[12]. One method to overcome these challenges due to high propagation losses is to use directive on-body antennas which focus the radiation towards the body, still meeting the Specific Absorption Ratio (SAR), and hence may strengthen the communication link between the implant and the on-body device [13]-[16]. The use of the antenna cavities is one commonly used approach to enhance directivity of the onbody antenna towards the body [13]-[15]. There are some studies on the channel characteristics between the capsule endoscope and directive antennas having different sizes of cavities [10]-[12], [16]. The studies are conducted in different capsule locations but only with two different on-body antenna locations. The results show that the selected two on-body antenna locations do not cover the intestine area fully and hence several on-body antennas are required to enable reliable communication link between the capsule and the on-body device thoroughly the whole intestine tract. Besides, multiantenna system is essential for several capsule localization techniques [17].

This study presents the radio channel evaluations between the capsule endoscope with five selected type of directive receiving on-body antennas in different capsule locations including the most challenging locations deep inside the tissues or far away from the most of the on-body antennas. Also, the easiest location is considered as well for the comparison. The goal is to compare the channel attenuation between the capsule and different on-body antennas and understand the reason for variations. Furthermore, the aim is to verify whether five of the selected type of directive on-body antennas can provide full coverage on the small intestine area.

The novelty of this research is that, up to author's knowledge, other UWB capsule endoscope studies do not evaluate radio channels with an anatomical voxel model and multiple directive on-body antennas. Besides, other studies do not provide discussion for channel attenuation variation by taking into the account the antenna's on-body radiation patterns and also the diverse propagation environment inside the tissues in the voxel model. 
The study is carried out with Simulia CST Studio Suite [18] and its anatomical voxel model Laura. A simplified capsule model used e.g. in [10]-[12] is used in the evaluations. Besides, a directive on-body antenna designed for low-band UWB in-body communications [15] for the frequency range 3.75-4.25 GHz according to IEEE 802.15.6 Wireless Body Area Networks (WBAN) standard [18], is used in this study.

This paper is organized as follows: Section II presents the study case describing the simulation model, on-body antenna, and the capsule endoscope model. Channel evaluations are presented in Section III for different capsule locations. Summary and Conclusions are given in Section V.

\section{STUDY CASE}

\section{A. Simulation model}

The simulations were conducted using finite integration technique (FIT) -based 3D electromagnetic simulation tool CST Studio Suite [19]. The basic idea of FIT is to calculate the radio propagation by solving the Maxwell's equations in their integral form [20].

The study is carried out using a female voxel model Laura which is presented in Fig. 1a. Laura resembles young lean female and it is chosen since its intestinal area has realistic shape. The coordinate system used in this study is included in Fig. 1 as well. In order to reduce computational complexity, the simulations are conducted covering only the abdominal part of Laura, as depicted in Fig. 1b, which covers the full size of the voxel model in $\mathrm{x}$ - and $\mathrm{y}$-directions but limiting from the $z$-direction to the intestine area which is $35 \mathrm{~cm}$ in Laura's case. Fig. 1b illustrates also the locations of the onbody antennas as well as one of the positions of the capsule endoscope which all are described more in detail in the following section. The black line in Fig. 1a presents the level for Laura's cross-section shown in Fig. 2. The cross-section illustrates the most relevant tissues for this study.

This paper focuses on analysis of frequency and time domain channel characteristics. Frequency domain channel characteristics, i.e. channel parameter S21, is obtained directly from CST as its complex form for the simulated frequency range $0.5-5 \mathrm{~dB}$. The frequency range of interest is $3.75-4.25 \mathrm{GHz}$, which is the mandatory channel in IEEE 802.15.6 standard for WBAN and for which the directive onbody antennas are designed. Time domain channel data is obtained by performing Inverse fast Fourier transform (IFFT) for S21, which has been root raised cosine windowed in the way that the flat area of the window is for the range 3.75-4.25 $\mathrm{GHz}$ and the cutoff frequencies are $3 \mathrm{GHz}$ and $5 \mathrm{GHz}$.

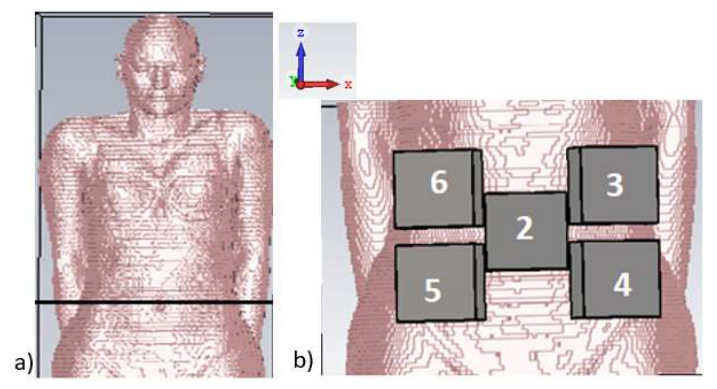

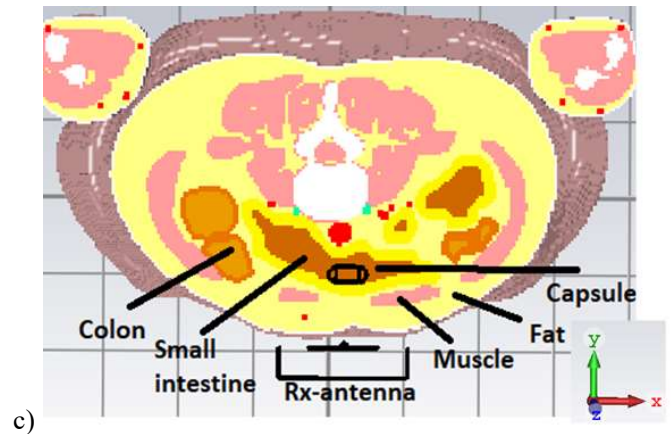

Fig. 1. a) Laura voxel model, b) abdominal area of torso selected for simulations, c) cross-section of the voxel model.

\section{B. On-body antenna}

In this study, we use a directive on-body antenna designed for in-body communications for low-UWB frequency range $3.75-4.25 \mathrm{GHz}$. The antenna, which is illustrated in Fig. 2a, is originally presented in [15] and it is later used in several in-body channel studies in [10]-[12]. Realized gains of the antenna as it is located on the body, are presented in Figs. 2ac for frequencies $3.75 \mathrm{GHz}, 4 \mathrm{GHz}$, and $4.25 \mathrm{GHz}$, which are the start, center and end frequencies of the range of interest, respectively. One should note that in this figure, the orientation of the coordinates is different to those presented in the voxel model cross-cuts since this coordinate orientation visualizes better the gain towards the body.

a)
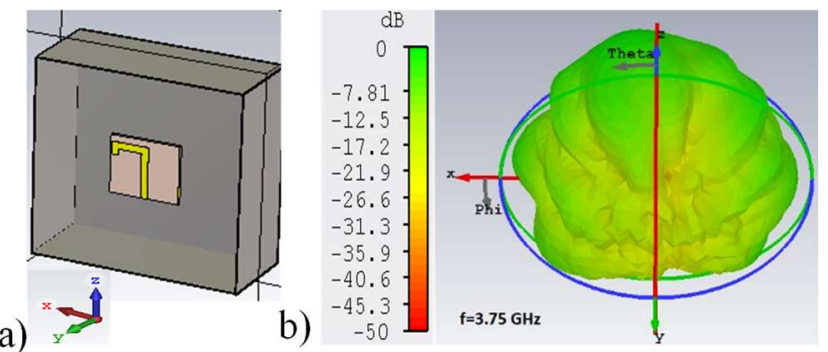

c)
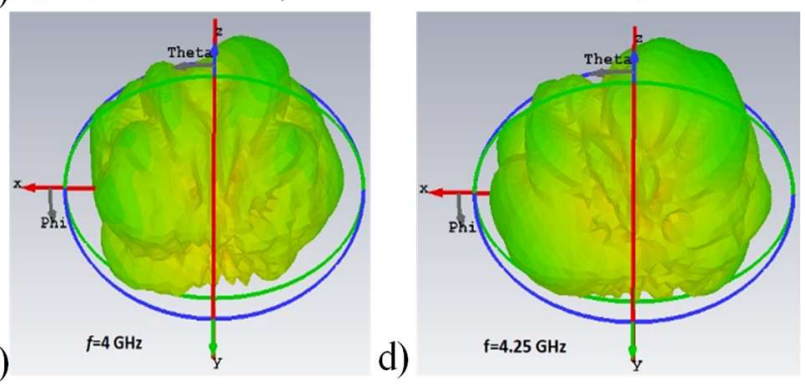

Fig. 2. a) The directive on-body antenna designed for in-body communications, b) realized gain at $3.75 \mathrm{GHz}$, c) realized gain at $4 \mathrm{GHz}$, realized gain at $4.25 \mathrm{GHz}$.

\section{Capsule model}

In this study, we use same simplified capsule model as e.g. in [10], in which details of the model can be found. In the capsule model, an omnidirectional dipole antenna is embedded in plastic capsule shell, which has realistic dimensions: $11 \mathrm{~mm}$ x $25 \mathrm{~mm}$, corresponding to the size of the commercial capsules nowadays [1]. The scheme of the dipole antenna and the capsule shell are presented in Fig. 3a-b, respectively. The dipole antenna is designed to work at the frequency of $4 \mathrm{GHz}$ inside the small intestine. 
a)
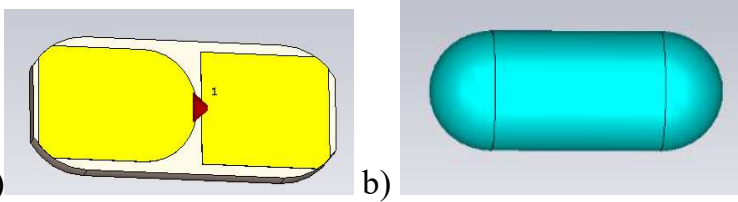

Fig. 3. a) Dipole antenna inside the capsule, b) and the capsule shell.

\section{Locations of on-body antennas and capsule endoscope}

In this study, we used five directive on-body antennas located on the voxel's abdomen area as was illustrated in Fig. 1b. Five on-body antennas were initially estimated to provide full coverage over the small intestine area based on power flow studies presented in [9] and [15]. Hence, the number of antennas was set to five for the studies presented in this paper. The antennas are numbered according to their port number in the simulation model, port number 1 is the antenna port of the capsule antenna, and port numbers 2-6 for the on-body antennas. The distance between the on-body antenna and the skin is approximately $4 \mathrm{~mm}$, which corresponds to the thin cloth thickness. Due to the pixelization of the voxel models, size of the antenna and shape of the voxel model, the antennabody distance may be slightly larger is some antenna locations which may have impact on the propagation depth as discussed in [21].

The channels between the capsule and the on-body antennas is evaluated in three different capsule locations. The capsule locations, presented in Figs.4a-c, are chosen to resemble the easiest and the most challenging locations in the small intestine area. In this study, the orientation of the capsule is kept fixed in all capsule locations. The impact of the capsule rotation can be studied e.g. in [12].

The location "A" is the middle of the abdomen is obviously the easiest location since it is close to the skin surface above which the on-body antenna 2 is located. Besides, the view from the other on-body antenna is relatively good.

The location " $\mathrm{B}$ " is the innermost corner in the right of the small intestine area. The challenge in this location is that it is far away even from the on-body antenna 3. However, there is a clear fat propagation path from this capsule location towards the on-body antenna 3.

The location " $\mathrm{C}$ " is the lowest part of the small intestine on the right. It is relatively close to the skin surface but there is colon area partially in front of the capsule. The capsule location is far from the other antennas except antenna 5. Thus, it is challenging to achieve strong channel between the capsule and several on-body antennas.

a)

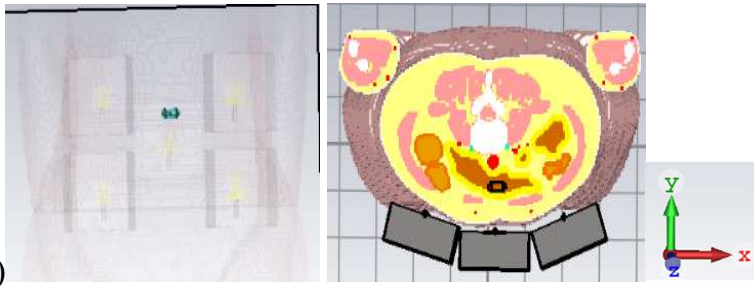

b)

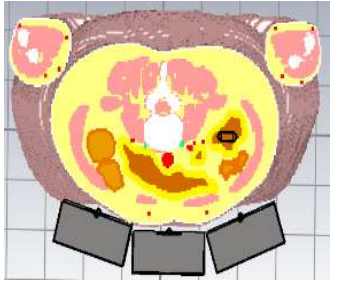

c)
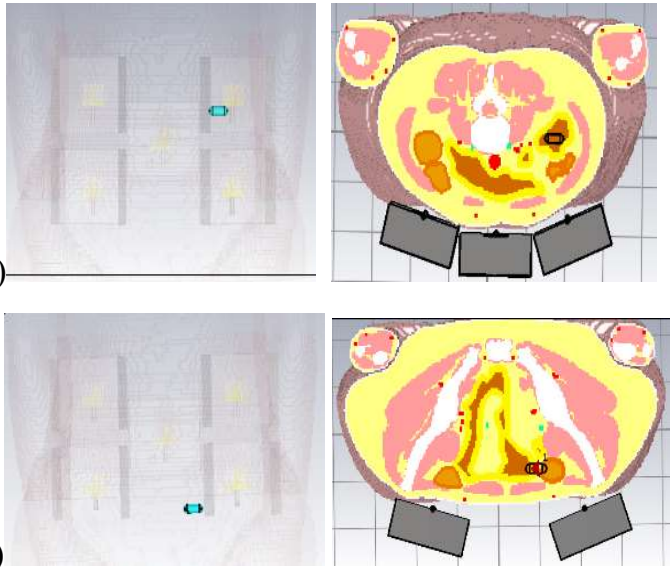

Fig. 4. Capsule locations a) "A", b) "B", c) "C"

\section{Channel EVAlUATIONS WITH FIVE ON-BODY ANTENNAS}

\section{A. Capsule location " $A$ "}

In this section, channel characteristics between the five on-body antennas and the capsule are evaluated in the capsule location "A". Frequency domain results, i.e. channel parameters S21, S31, S41, S51, and S61, as well as time domain results, impulse responses (IR)s IR21, IR31, IR41, IR51, and IR61, are presented in Figs.5a-b, respectively.

The frequency domain results are presented only for the frequency range of interest, i.e. $3.75-4.25 \mathrm{GHz}$. One can note clearly smaller channel attenuation between the capsule and the on-body antenna 2 than with the other on-body antennas. As noted from Fig. 4a, the capsule is located symmetrically between the on-body antennas 3 and 6 and hence the channel attenuation is somewhat similar to those antennas. Also, the on-body antennas 4 and 5 are located symmetrically respect to the capsule. However, the difference between S41 and S51 is more remarkable than that of S31 and S61. The maximum attenuation difference between S41 and $\mathrm{S} 51$ is $10 \mathrm{~dB}$, whereas between S31 and S61 it is $3 \mathrm{~dB}$. Similar tendency can be noted also in impulse responses (IR) presented in Fig. 5b. Propagation loss and propagation time are clearly smallest in the impulse response between the onbody antenna 2 and capsule (IR21) than in the other impulse responses (IR31-IR61). Also in time domain, IR between the capsule and the on-body antenna 3 is similar as that of the onbody antenna 6 . The level and width of the main peaks are almost equal, the only difference comes from the timing of the first side peaks. Instead, the difference between the IR for antennas 4 and 5 is more remarkable as expected from the frequency domain results. At the time range 2-6 ns, the IR41 is clearly at higher level. However, both channels are relatively weak which is understandable due to their locations.

Although the antennas 3 and 6 are positioned symmetrically respect to the capsule, the differences in the channel responses are due to the small asymmetries in the radiation patterns of the on-body antennas towards lower left and lower right parts as seen in Figs. 2 b-c. Especially at 4 $\mathrm{GHz}$, one can note clear asymmetry between the right and left parts. In the capsule location ' $A$ ', the on-body antenna 6 has stronger beam towards the capsule than the on-body antenna 3 . This asymmetry is even more evident between the upper 
left and upper right parts of the radiation patterns which affects on the channel between the capsule and the on-body antennas 4 and 5. For instance, at $3.75 \mathrm{GHz}$, channel attenuation is $10 \mathrm{~dB}$ larger for the antenna 5 than the antenna 4. When we study the radiation patterns at $3.75 \mathrm{GHz}$ in Fig. $2 \mathrm{a}$, we can see the clear notches in the upper left area, which is directly towards the capsule location ' $A$ ' with the on-body antenna 5 . Instead, there are relatively strong beams towards the upper right area in the radiation pattern, which is directly towards capsule location 'A' with the on-body antenna 4.

Furthermore, there is asymmetry in voxel model's tissues as seen from Fig. 4a: the voxel model's abdominal muscle is clearly smaller at this horizontal cross-cut level and hence the amount of fat is larger. Since the fat is known to be a good propagation medium [22]-[24], this is one reason for smaller channel attenuation for the on-body antenna 6 , which is located on the left side of the voxel model.

For accurate capsule localization, relevant channel strength is required for at least three on-body antennas [17]. For the capsule location "A", the channels for each on-body antenna are at reasonable level for capsule localization.

a)

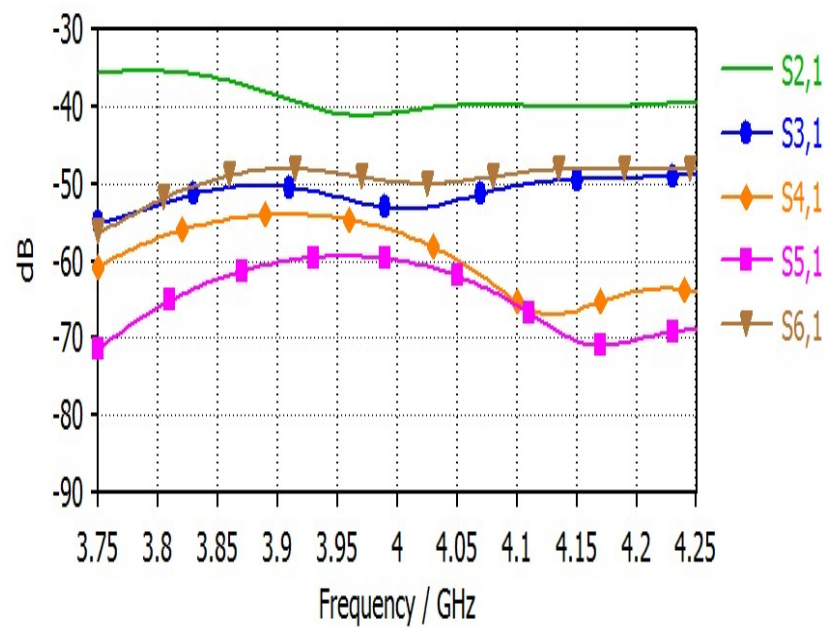

b)

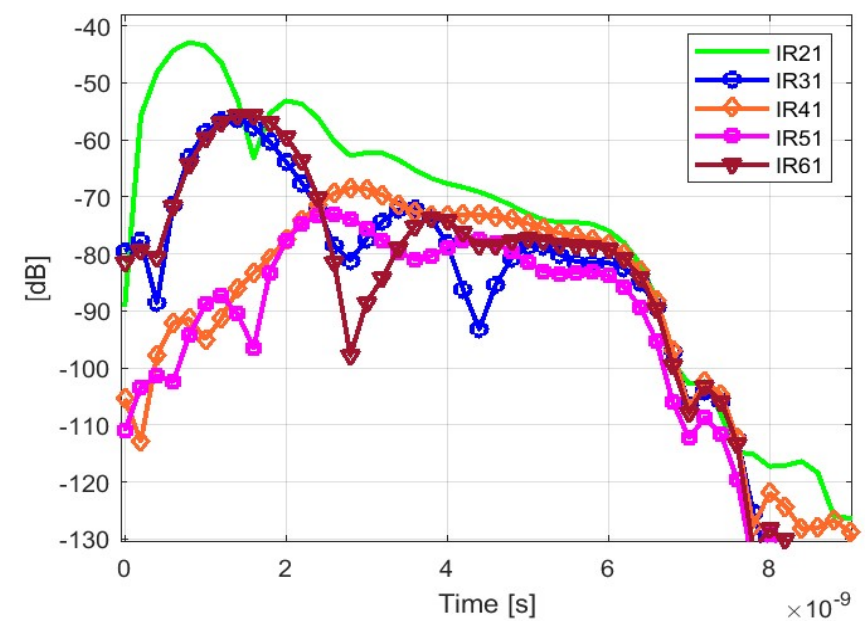

Fig. 5. Frequency and b) time domain channel characteristics at the antenna location "A".

\section{B. Capsule location "'B"}

In this section, channel characteristics between the onbody antennas and the capsule are evaluated in the capsule location " $b$ ", which is deep in the rightmost corner of the small intestine area. Frequency domain results S21-S61 and time domain results are presented in Figs.6a-b, respectively. In this case it is obvious that the smallest channel attenuation occurs between the capsule and the on-body antenna 3, for which the propagation distance is the smallest. However, the attenuation is anyway relatively high: $-70--80 \mathrm{~dB}$ for the frequency range of interest. For the on-body antenna 2 , the attenuation is below $-80 \mathrm{~dB}$, whereas for the rest of the channels the attenuation is close to $-100 \mathrm{~dB}$ or even more. Interestingly, channel S61 is strongly attenuated up to 3.97 $\mathrm{GHz}$, after which the channel strength increases clearly. With channel S51, it is vice versa: channel attenuation increases after $3.97 \mathrm{~dB}$. The reason for these changes can be found from radiation patterns. In time domain, the main peaks are at low level, varying from $-90 \mathrm{~dB}$ to $-130 \mathrm{~dB}$. However, the main and side peaks are clearly above the noise level and thus, the signal can be extracted in the receiver.

a)

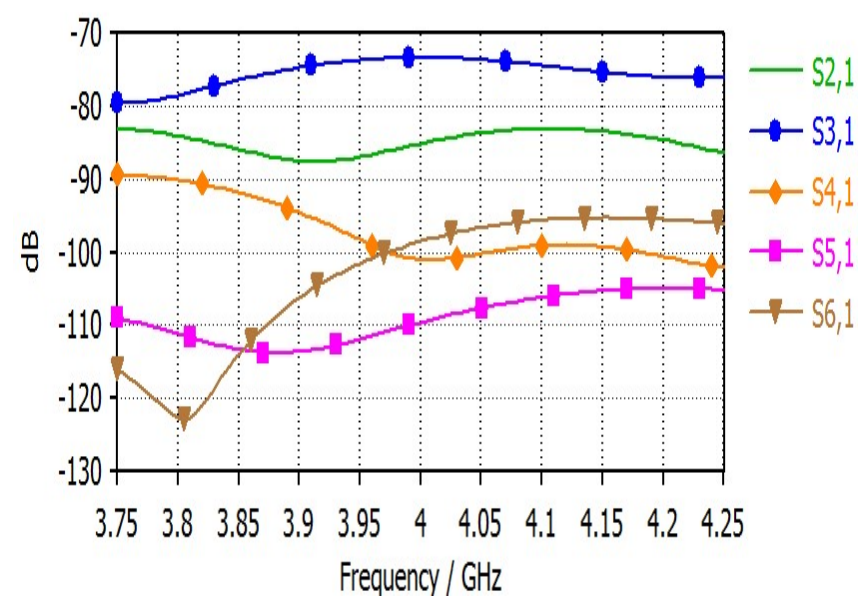

b)

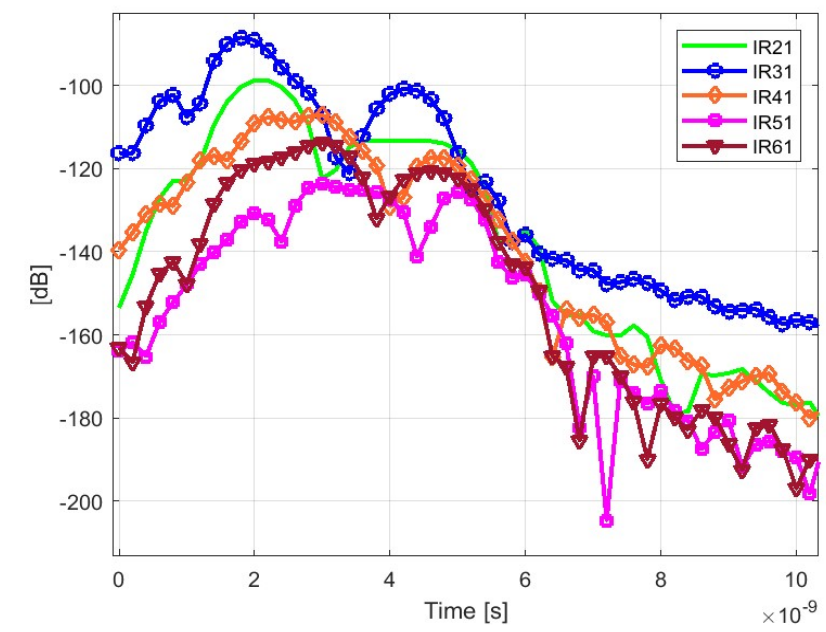

Fig. 6. a) Frequency and b) time domain channel characteristics at the antenna location "B" 
In the capsule location " $C$ ", which is the lowest part of the small intestine area, only the on-body antenna 4 is closely located to the capsule. The on-body antennas 2 and 6 are the next closest antennas for the capsule, whereas the antennas 3 and 6 are the furthest antennas. The channel responses presented in Figs. 7a-b are show interestingly the impact of the antenna radiation patterns and their changes to the channel attenuation at this capsule location. Evidently, S41 is at the highest level through the whole frequency range of interest due to its nearest location for the capsule. With the next closest antennas, 2 and 5, there is remarkable variation over the frequency range of interest. For S51, the attenuation is among the largest up to $3.87 \mathrm{GHz}$ after which attenuation diminishes and surprisingly achieves the level of S41 at 4.2 $\mathrm{GHz}$. Similar tendency is noted for S21 expect the channel attenuation decreases from $4 \mathrm{GHz}$ onwards. The channel attenuation is modest for the antenna 3 although it is relatively far away from the capsule. Besides of radiation pattern, also both visceral and outer fat tissues enhance propagation with less power loss [23]-[24]. From the capsule location $\mathrm{C}$, there is short distance to the outer fat layer thorough which the signal may propagate easily to the onbody antenna 3 . This is obviously reason for the moderate channel attenuation for the antenna 6 , which is the furthest antenna from the capsule.

In time domain results presented in Fig. $8 \mathrm{~b}$, one can note similar tendency. The difference in the level of the main and side peaks is relatively moderate between all the channels. Clearly stronger variation was seen e.g. in capsule location ' $\mathrm{B}$ '. The reason for this phenomenon is that in capsule location ' $\mathrm{B}$ ', which is deep inside the abdomen, fat as propagation medium though the outer fat layer can not be utilized similarly as in capsule location ' $C$ ', in which capsule is located close to the outer fat layer. As it has been shown in power flow studies in [24], the signal from the implant may propagate over the wide area on the surface of the abdomen, if it has smooth access though the visceral fat layers to the outer fat layers.

a)

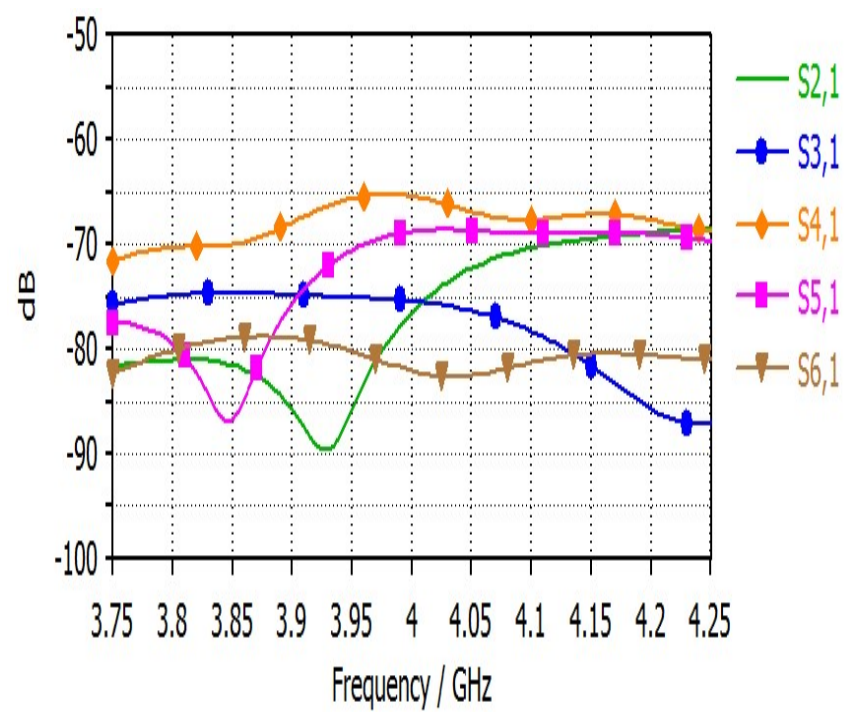

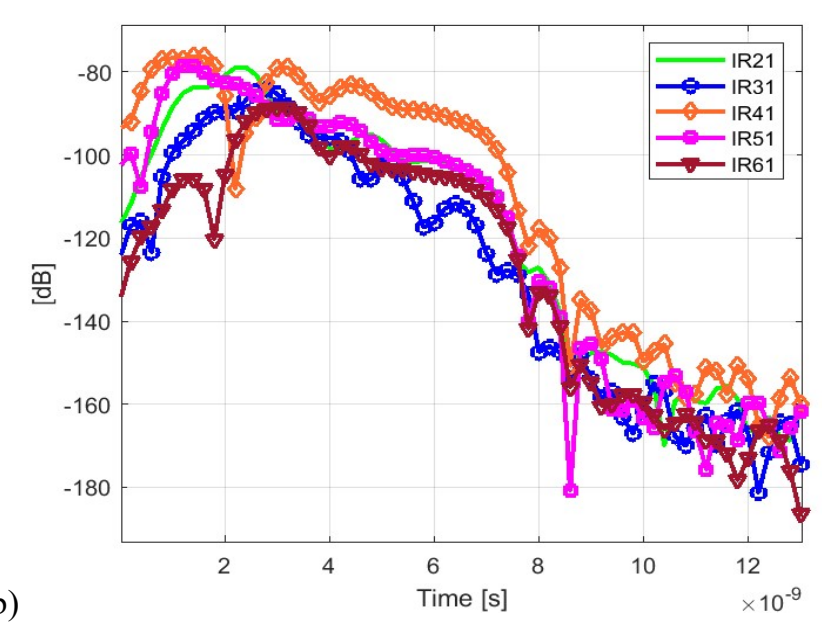

Fig. 7. Frequency and time domain channel characteristics in the capsule location "C".

\section{SUMMARY AND CONCLUSIONS}

This paper presented a study on channel characteristics between the capsule endoscope and multi on-body antenna system. The aim was to study the variation of the frequency and time domain channel characteristics for the on-body antennas in different capsule locations, including the most challenging capsule locations deep inside the tissues or far away from the most of the antennas.

It was found that five of the selected type of directive onbody antennas provide sufficient coverage over the intestine area even in the most challenging locations. In the "easiest" location, i.e. the capsule is located in the middle of the antenna configuration close to the visceral fat layer, the channel attenuation is modest for all of the antennas. In such situation, communication link between the capsule and onbody device is reliable as well as localization of the capsule easy. If the capsule was located deep inside the tissues, the variation between the channel attenuations can be significant, over $40 \mathrm{~dB}$ within the frequency range of interest. This is due to lack of smooth access from the capsule to the subcutaneous fat layer via the visceral fat layer, through which the signal could travel easily to different on-body antennas. Instead, if the capsule was located close to the visceral fat layer, the channel attenuation was found to be moderate even for the antennas which are located far from the capsule. However, also in these challenging locations, IR peaks clearly rises from the noise level.

Multiple on-body antennas are required to provide reliable communication link between the capsule and the onbody device thoughout whole intestine tract. Besides, multiantenna system is also essential for several capsule localization techniques. Thus, it is important to study signal propagation from the capsule towards the on-body antennas using voxel models which anatomically resemble human intestine areas. This study was conducted using one type of directive on-body antenna which has been used in several inbody propagation studies. The number of receive on-body antennas required for sufficient coverage over the intestine area depends on the antenna types and their radiation characteristics. Our next target is to extend this study to cover several different on-body antennas designed for inbody communications with different types of 
cavities/reflectors and without reflector as well as determine optimal number for each antenna type. Besides, the future work will include evaluations with different voxel models having different sizes and body constitutions.

\section{ACKNOWLEDGMENT}

This work is supported in part by the projects WBAN Communications in the Congested Environments (MeCCE), the Academy of Finland 6Genesis Flagship (grant 318927) and the European Union's Horizon 2020 programme under the Marie Sklodowska-Curie grant agreement No. 872752. Doc. Matti Hämäläinen is acknowledged for project management. Dr. Markus Berg is acknowledged for capsule antenna design. Dr. Marko Sonkki is acknowledged for his participation on the on-body antenna design.

\section{REFERENCES}

[1] G. Ciuti; A. Menciassi; P. Dario, "Capsule Endoscopy: From Current Achievements to Open Challenges", IEEE Reviews in Biomedical Engineering, vol.4, pp. 59-72, 2011.

[2] D. R. Cave, S. Hakimian, and K. Patel, "Current controversies concerning capsule endoscopy," Digestive Diseases and Sciences, vol. 64, no. 11, pp. 3040-3047, 2019.

[3] H. Neumann, L. C. Fry, A. Na"gela, and M. F. Neurath ; "Wireless capsule endoscopy of the small intestine: a review with future directions"; Current Opinion in Gastroenterology; Vol 30; Issue 5; pp 463-471, 2014.

[4] R. Chavez-Santiago, J. Wang, and I. Balasingham, "The Ultra Wideband Capsule Endoscope," International Conf. on Ultra Wideband, 2013.

[5] https://www.itis.ethz.ch/virtual-population/tissue-properties/database

[6] C. Garcia-Pardo, M. Barbi, S. Perez-Simbor and N. Cardona, "UWB Channel Characterization for Wireless Capsule Endoscopy Localization," 2020 IEEE International Conference on Communications Workshops (ICC Workshops), Dublin, Ireland, 2020.

[7] J.-C. Brumm, H. Strohm, and G. Bauch, "A stochastic channel model for ultra wideband in-body communication," 201941 st Annual International Conference of the IEEE Engineering in Medicine and Biology Society (EMBC). IEEE, 2019.

[8] X. Fang, M. Ramzan, Q. Zhang, S. Perez-Simbor, S. Wang, N. Neumann, and D. Plettemeier, "Experimental In-Body to On-Body and In-Body to In-Body Path Loss Models of Planar Elliptical Ring Implanted Antenna in the Ultra-Wide Band," 2019 13th International Symposium on Medical Information and Communication Technology (ISMICT). IEEE, 2019.

[9] C. Garcia-Pardo, A. Fornes-Leal, N. Cardona, R. Chavez-Santiago, J. Bergland, I. Balasingham, S. Brovoll, O. Aardal, S. Hamran, and R. Palomar, "Experimental ultra wideband path loss models for implant communications," 2016 IEEE 27th Annual International Symposium on Personal, Indoor, and Mobile Radio Communications (PIMRC). IEEE, 2016.

[10] M. Särestöniemi, C. Pomalaza Raez., C. Kissi, M. Berg, M. Hämäläinen, J. Iinatti, "WBAN channel characteristics between capsule endoscope and receiving directive UWB on-body antennas", IEEE Access Special Session on Body Area Networks, March 2020.

[11] M. Särestöniemi, C. Pomalaza-Raez, M. Berg, C. Kissi, M Hämäläinen, J. Iinatti, "In-Body Power Distribution for Abdominal Monitoring and Implant Communications Systems," ISWCS, September 2019.

[12] M. Särestöniemi, C. Pomalaza-Raez, M. Berg, C. Kissi, M. Hämäläinen, J. Iinatti, "UWB-WBAN Radio Channel Characteristics between the Endoscope Capsule and On-body Antenna," Bodynets2019, October 2019

[13] Q. V. d. Brande; S. Lemey; J. Vanfleteren; and Hendrik Rogier, "Highly-Efficient Impulse-Radio Ultra-Wideband Cavity-Backed Slot Antenna in Stacked Air-Filled Substrate-Integrated-Waveguide Technology”, IEEE Transactions on Antennas and Propagation, vol. 66, pp. $2199-2209,2018$.
[14] R. Ferreira; J. Joubert; and J. W. Odendaal, “A compact dual-circularly polarized cavity-backed ring-slot antenna", IEEE Transactions on Antennas and Propagation, vol. 65, pp. 364 - 368, 2017.

[15] C. Kissi, M. Särestöniemi, C. Pomalaza-Raez, M. Sonkki, M. N. Srifi, "Low-UWB directive antenna for Wireless Capsule Endoscopy Localization," BodyNets 2018.

[16] M. Särestöniemi, C. Pomalaza-Raez, C. Kissi, M. Berg, M. Hämäläinen, J. Iinatti, "Impact of the antenna cavity on in-body propagation and channel characteristics between capsule endoscope and on-body antenna," International Symposium on Medical Information and Technology (ISMICT), May 2020.

[17] H. Mateen, R. Basar, A. U. Ahmed and M. Y. Ahmad, "Localization of Wireless Capsule Endoscope: A Systematic Review," in IEEE Sensors Journal, vol. 17, no. 5, pp. 1197-1206, 1 March1, 2017, doi: 10.1109/JSEN.2016.2645945.

[18] IEEE Standard for Local and metropolitan area networks _Part 15.6: Wireless Body Area Networks, pp. IEEE Std 802.15.6-2012, pp. 1 271, 2012.

[19] CST Studio Suite, [Online]. Available: http://www.cst.com

[20] S. J. Orfanidis, "Electromagnetic Waves and Antennas," http://www.ece.rutgers.edu/ orfanidi/ewa/.

[21] M. Särestöniemi, C. Kissi, C. Pomalaza Raez. M. Hämäläinen, J. Iinatti, "Impact of the Antenna-Body Distance on WBAN Channel Characteristics," ISMICT2019, May 2019

[22] N. B. Asan, E. Hassan, J. Shah, D. Noreland, T. Blokhuis, R. Augustine, "Characterization of the fat channel for intra-body communication at R-band frequencies," Sensors, vol. 18, no. 9, p. 2752,2018

[23] M. Särestöniemi, C. Pomalaza-Raez, M. Berg, C. Kissi, M. Hämäläinen. J. Iinatti, "Fat in the Abdomen as a Propagation Medium in WBAN Applications," Bodynets2019.

[24] M. Särestöniemi, C. Pomalaza-Raez, C. Kissi, J. Iinatti, "Simulation and Measurement data based study on fat as propagation medium in WBAN abdominal implant communication system," accepted to be published in IEEE Access, March 2021. 